\title{
A new pressure-parametrization unified dark fluid model
}

\author{
Deng Wang ${ }^{1, \mathrm{a}}$, Yang-Jie Yan ${ }^{2, \mathrm{~b}}$, Xin-He Meng ${ }^{2, \mathrm{c}}$ \\ ${ }^{1}$ Theoretical Physics Division, Chern Institute of Mathematics, Nankai University, Tianjin 300071, China \\ ${ }^{2}$ Department of Physics, Nankai University, Tianjin 300071, China
}

Received: 14 January 2017 / Accepted: 12 April 2017 / Published online: 26 April 2017

(C) The Author(s) 2017. This article is an open access publication

\begin{abstract}
We propose a new pressure-parametrization model to explain the accelerated expansion of the late-time Universe by considering the baryon matter and dark contents (dark matter and dark energy) as a unified dark fluid. To realize this model more physically, we reconstruct it with the quintessence and phantom scalar fields, respectively. We use the recent cosmological data to constrain this model, distinguish it from the standard cosmological model and find that the value of the Hubble constant $H_{0}=68.34_{-0.92}^{+0.53}$ supports the global measurement by the Planck satellite at the $1 \sigma$ confidence level.
\end{abstract}

\section{Introduction}

With the ever-growing data, modern cosmological observations such as Type Ia supernovae ( $\mathrm{SNe}$ Ia), baryonic acoustic oscillations (BAO), cosmic microwave background (CMB) anisotropies, observational Hubble parameter $(H(z))$, etc., have confirmed the fact that our Universe is undergoing a phase of accelerated expansion [1-4]. The best way to explain such an evolution is the simple addition of the cosmological constant (CC) or $\Lambda$ term [5] in the framework of general relativity (GR). However, this otherwise formally and observationally consistent model meets two unsolved puzzles, i.e., the so-called fine-tuning and coincidence problems [6]. The former indicates that the enormous disagreement between the energy scale introduced by the $\mathrm{CC}$ and the predictions of standard model of particle physics for the vacuum energy density, while the latter, which implies the $\Lambda$-cold-dark-matter $(\Lambda \mathrm{CDM})$ model, cannot explain why the Universe is only recently accelerating. As a consequence, to alleviate or even solve these problems, a flood of alternative cosmological models based on different physical origins are proposed and

\footnotetext{
a e-mail: Cstar@mail.nankai.edu.cn

be-mail: yanyj926@gmail.com

c e-mail: xhm@nankai.edu.cn
}

studied by cosmologists. In the literature, there are mainly two classes of models, i.e., the well-known dark energy (DE) models, which introduce a new matter component in the cosmic pie, and the theories of modified gravities (MOG), which deviate from the standard GR. Here we only list a part of them as follows: phantom [7], quintessence [8-15], bulk viscosity [16-21], decaying vacuum [22-24], Chaplygin gas [25], $\mathrm{f}(\mathrm{R})$ gravity [26-31], Einstein-aether gravity [32,33], braneworld gravity [34-36] and so on.

As is well known, the parametrization method can be regarded as a powerful and useful tool to characterize the properties of the DE component in the late-time Universe. This method has been widely applied into confronting cosmological observations in recent years. Generally speaking, to investigate the properties and features of DE component, the mainstream of the DE parametrization is the equation of state (EoS) parametrization. One can express this class of parametrization in a polynomial form $\omega(z)=$ $\sum_{n=0} \omega_{n} x_{n}(z)$, where the expansions could be provided as follows: (i) $x_{n}(z)=z^{n}$; (ii) $x_{n}(z)=\left(\frac{z}{1+z}\right)^{n}$; (iii) $x_{n}(z)=$ $[\ln (1+z)]^{n}$; (iv) $x_{n}(z)=\left[\frac{z(1+z)}{1+z^{2}}\right]^{n}$, etc. The parametrization (i) was firstly proposed by Huterer and Turner [37], and Weller and Albrecht [38] investigate the case of $n \leqslant 1$. Although this parametrization behaves very well at low redshifts, it exhibits a problematic behavior at high redshifts such as failing to explain the age estimations of high $-z$ ( $z$ denotes the redshift) objects, since it predicts substantially small ages at $z \geqslant 3$. The so-called Chevalier-Polarski-Linder (CPL) parametrization $[39,40]$ (ii) with the case of $n \leqslant 1$ is dedicated to solving the above-mentioned problematic behavior at high redshifts, which fits well for a great deal of theoretically conceivable scalar field potential and provides an excellent explanation for small deviations from the phantom barrier $(\omega=-1)$ [41]. The parametrization (iii) with the case of $n \leqslant 1$ was introduced by Efstathiou [42], which is intended to adjust some quintessence-like scenarios at $z \lesssim 4$. The parametrization (iv) was proposed by Barboza et al. [43], which is aimed at extending the range of applicability of 
the DE EoS, and avoid the uncertainties and singularities in the aforementioned three scenarios. Recently, several new parameterizations have been proposed, such as applying the Padé method into the DE EoS [44].

About 10 years ago, Sen proposed a parametric scenario for the effective pressure of the DE, namely $P_{\Lambda}=$ $-P_{0}+P_{1}(1-a)+\cdots$ so as to study the small deviations from the $\triangle \mathrm{CDM}$ model [45], where $a$ is the scale factor. Following this theoretical line, Zhang et al. [46] have developed two parametric models for the effective pressure of the unified dark fluid (UDF), i.e., $P(z)=P_{a}+P_{b} z$ and $P(z)=P_{c}+\frac{P_{d}}{1+z}$, and they found these two models fit current observations well. The two scenarios both indicate the DE EoS $\omega<-1$, which corresponds to a phantom-like case at the present epoch. Actually, one can easily find that this class of parametrization is equivalent to the above-mentioned class through some simple derivations. In this situation, to continue studying the underlying DE model deviating from the $\Lambda \mathrm{CDM}$ model, we propose a new unified pressure-parametrization dark fluid model, investigate its evolutional behavior, place constraints on it using the recent cosmological data, and explore the corresponding value of the Hubble constant $H_{0}$.

looseness-1The rest of this study is organized in the following manner. In the next section, we propose the new model. In Sect. 3, we reconstruct it with quintessence and phantom scalar fields, respectively. In Sect. 4, we constrain this model by using the recent cosmological observations. In Sect. 5, we distinguish this model from the $\triangle \mathrm{CDM}$ model. In Sect. 6, we investigate the $H_{0}$ value of our model. The discussions and conclusions are presented in the final section (we take units $8 \pi G=c=1$ ).

\section{The model}

The Friedmann equations, the conservation equation of the stress-energy tensor, and the EoS compose a close dynamical system to characterize the background evolution of the Universe. As mentioned above, the EoS is equivalent to the relation between the effective pressure and the redshift. For a spatially flat Friedmann-Robertson-Walker (FRW) spacetime, on the one hand, one can obtain the $P(z)-z$ relation by inserting the EoS into the equation of energy conservation. On the other hand, the EoS can be recovered by inserting the $P(z)-z$ relation into the equation of energy conservation, which is expressed as

$\dot{\rho}+3 H(\rho+P)=0$.

Notice that the $\triangle \mathrm{CDM}$ scenario is not strictly supported by current cosmological observations since there exists a small deviation. However, the physical mechanism of the small deviation is still not clear. Different from the scenarios including an extra component and the evolution of the
EoS, we would like to regard the dark content in the latetime Universe as a UDF, and investigate the parametrization scenario for effective pressure of the UDF which actually circumvents the issue of possible physical mechanism. Utilizing this approach, we can study the possible deviations from the constant case of the $P(z)-z$ relation without a specific presupposition.

The new pressure-parametrization scenario as a UDF can be written as

$P(z)=P_{a}+P_{b}\left(z+\frac{z}{1+z}\right)$,

where $P_{a}$ and $P_{b}$ are two different free parameters. Replacing Eq. (2) in Eq. (1), we have

$\rho(a)=-P_{a}+\frac{3}{4} P_{b}\left(a-2 a^{-1}\right)+C a^{-3}$,

where $C=\rho_{0}+P_{a}+\frac{3 P_{b}}{4}$ is an integration constant and $\rho_{0}$ is the present-day energy density. Note that the subscript " 0 " represents the present values of the corresponding physical quantities and we have used the usual relation $a=\frac{a_{0}}{1+z}=$ $\frac{1}{1+z}$ here. It is interesting to see that in Eq. (3), the constant term $P_{a}$ and the last term $\mathrm{Ca}^{-3}$ can be regarded as the $\mathrm{CC}$ term and the dust matter term (baryons and dark matter) in the $\Lambda \mathrm{CDM}$ model, respectively. The second term $\frac{3}{4} P_{b}(a-$ $2 a^{-1}$ ) can be interpreted as the small deviations from the CC.

Subsequently, dividing both sides of Eq. (3) by $\rho_{0}$ and using the first Friedmann equation, $H^{2}=\frac{\rho}{3}$, we obtain the dimensionless Hubble parameter:

$E(a)=\left\{\alpha+\beta\left(2 a^{-1}-a\right)+(1-\alpha-\beta) a^{-3}\right\}^{\frac{1}{2}}$,

where $E(a)=H(a) / H_{0}$, and $\alpha=-P_{a}^{*}$ and $\beta=-\frac{3}{4} P_{b}^{*}$ $\left(P_{a}^{*}=P_{a} / \rho_{0}, P_{b}^{*}=P_{b} / \rho_{0}\right)$ are two free parameters to be constrained by observations in the following section. To exhibit the scalar field reconstructions of our model better, we derive the EoS of our model as follows:

$\omega_{\mathrm{de}}=-1+\frac{\beta\left(2 a^{-1}+a\right)}{3\left[\alpha+\beta\left(2 a^{-1}-a\right)\right]}$.

\section{The reconstructions}

In principle, the deviations from the $\Lambda \mathrm{CDM}$ in our model can be realized by different physical scenarios. The scalar field reconstructions are main methods to explain the accelerated expansion of the Universe at the present epoch. To perform a detailed analysis in the context of reconstructions, one must investigate the evolutional behaviors of the scalar fields and potential over cosmic history, and compare them with those of energy density and pressure of our pressureparametrization UDF model at low redshifts. The specific 
realization of the scalar field reconstructions can be expressed as

$P_{\text {eff }}=P_{\text {scalar }}$

$\rho_{\text {eff }}=\rho_{\text {scalar }}+\rho_{m}$,

where $\rho_{m}$ is the matter density in the late-time Universe. In this work, we would like to reconstruct our model with quintessence and phantom scalar fields, respectively.

The quintessence $\left(\omega_{\mathrm{de}}>-1\right)$ corresponds to a canonical scalar field $\phi$ with a potential $V(\phi)$ that only interacts with the standard gravity, and its action is written as

$S=\int \mathrm{d}^{4} x \sqrt{-g}\left[\frac{R}{2}-\frac{1}{2} g^{\mu \nu} \partial_{\mu} \phi \partial_{\nu} \phi-V_{1}(\phi)\right]+S_{m}$,

where $R$ is the Ricci scalar and $S_{m}$ is the action of matter. By the variation of the lagrangian of quintessence with respect to (w.r.t.) $\phi$, one can obtain

$\ddot{\phi}+3 H \dot{\phi}+V_{1}^{\prime}(\phi)=0$,

where the dot and the prime denote the derivatives w.r.t. the cosmic time and the scalar field $\phi$, respectively. In a spatially flat FRW Universe, the energy density and pressure of the quintessence scalar field can be expressed as

$\rho_{\mathrm{de}}=\frac{1}{2} \dot{\phi}^{2}+V_{1}(\phi)$

$P_{\mathrm{de}}=\frac{1}{2} \dot{\phi}^{2}-V_{1}(\phi)$

It follows that the EoS is

$\omega_{\mathrm{de}}=\frac{\frac{1}{2} \dot{\phi}^{2}-V_{1}(\phi)}{\frac{1}{2} \dot{\phi}^{2}+V_{1}(\phi)}$.

The phantom corresponds to a scalar field which is minimally coupled to the standard gravity and matter sources, and its action is

$S=\int \mathrm{d}^{4} x \sqrt{-g}\left[\frac{R}{2}+\frac{1}{2} g^{\mu \nu} \partial_{\mu} \phi \partial_{\nu} \phi-V_{2}(\phi)\right]+S_{m}$.

The dynamically evolutional equation of the phantom field can be written as

$\ddot{\phi}+3 H \dot{\phi}-V_{2}^{\prime}(\phi)=0$.
Furthermore, the energy density, pressure and EoS of the phantom field can be, respectively, written

$\rho_{\mathrm{de}}=-\frac{1}{2} \dot{\phi}^{2}+V_{2}(\phi)$

$P_{\mathrm{de}}=-\frac{1}{2} \dot{\phi}^{2}-V_{2}(\phi)$

$\omega_{\mathrm{de}}=\frac{-\frac{1}{2} \dot{\phi}^{2}-V_{2}(\phi)}{-\frac{1}{2} \dot{\phi}^{2}+V_{2}(\phi)}$.

\subsection{The quintessence case}

Assuming that the cosmic pie consists of two ingredients (quintessence and matter) and using Eqs. (2), (3) and (15), (16), one can easily obtain

$\frac{1}{2} \dot{\phi}^{2}+V_{1}(\phi)=-P_{a}+\frac{3}{4} P_{b}\left(a-2 a^{-1}\right)$,

$\frac{1}{2} \dot{\phi}^{2}-V_{1}(\phi)=P_{a}+P_{b}\left(a^{-1}-a\right)$.

Simplifying the above two equations with the redefined parameters $\alpha$ and $\beta$, we have

$\frac{1}{2} \dot{\phi}^{2}=\frac{1}{6} \rho_{0} \beta\left(a+2 a^{-1}\right)$,

$V_{1}(\phi)=\rho_{0}\left[\alpha+\frac{1}{6} \beta\left(10 a^{-1}-7 a\right)\right]$.

In order to solve Eqs. (20) and (21) conveniently, we use the initial condition $\phi_{a=1}=M_{\mathrm{pl}}$, where $M_{\mathrm{pl}}$ is the reduced Planck mass. Then the first Friedmann equations can be rewritten as $H^{2}=\frac{1}{3 M_{\mathrm{pl}}} \rho$. Considering the dark energy dominated Universe at the present stage with the density ratio parameter $\Omega_{\mathrm{de}} \sim 0.7$, we can define the present value of the potential as $V_{0}=\rho_{0}=3 M_{\mathrm{pl}}^{2} H_{0}^{2}$. Subsequently, by solving Eqs. (20) and (21), one can obtain

$\frac{\mathrm{d} \phi}{\mathrm{d} a}= \pm M_{\mathrm{pl}}\left[\frac{\beta\left(a^{-1}+2 a^{-3}\right)}{\alpha+\beta\left(2 a^{-1}-a\right)+(1-\alpha-\beta) a^{-3}}\right]^{\frac{1}{2}}$,

$V_{1}(\phi)=V_{0}\left[\alpha+\frac{1}{6} \beta\left(10 a^{-1}-7 a\right)\right]$,

where the symbol " \pm " denotes two different solutions. We would like to study the evolutional behaviors of the quintessence fields numerically by considering $\alpha=0.7$ and $\beta=0.02$. The results are presented in Fig. 1. One can find that $\phi$ increases with the increasing scale factor $a$ in the upper left panel of Fig. 1, and that the potential decreases monotonically with the increasing $\phi$ and it will reach the minimal value at the recent future $z \approx$ $-5.3 \times 10^{-10}$ (see the lower left panel of Fig. 1), which 

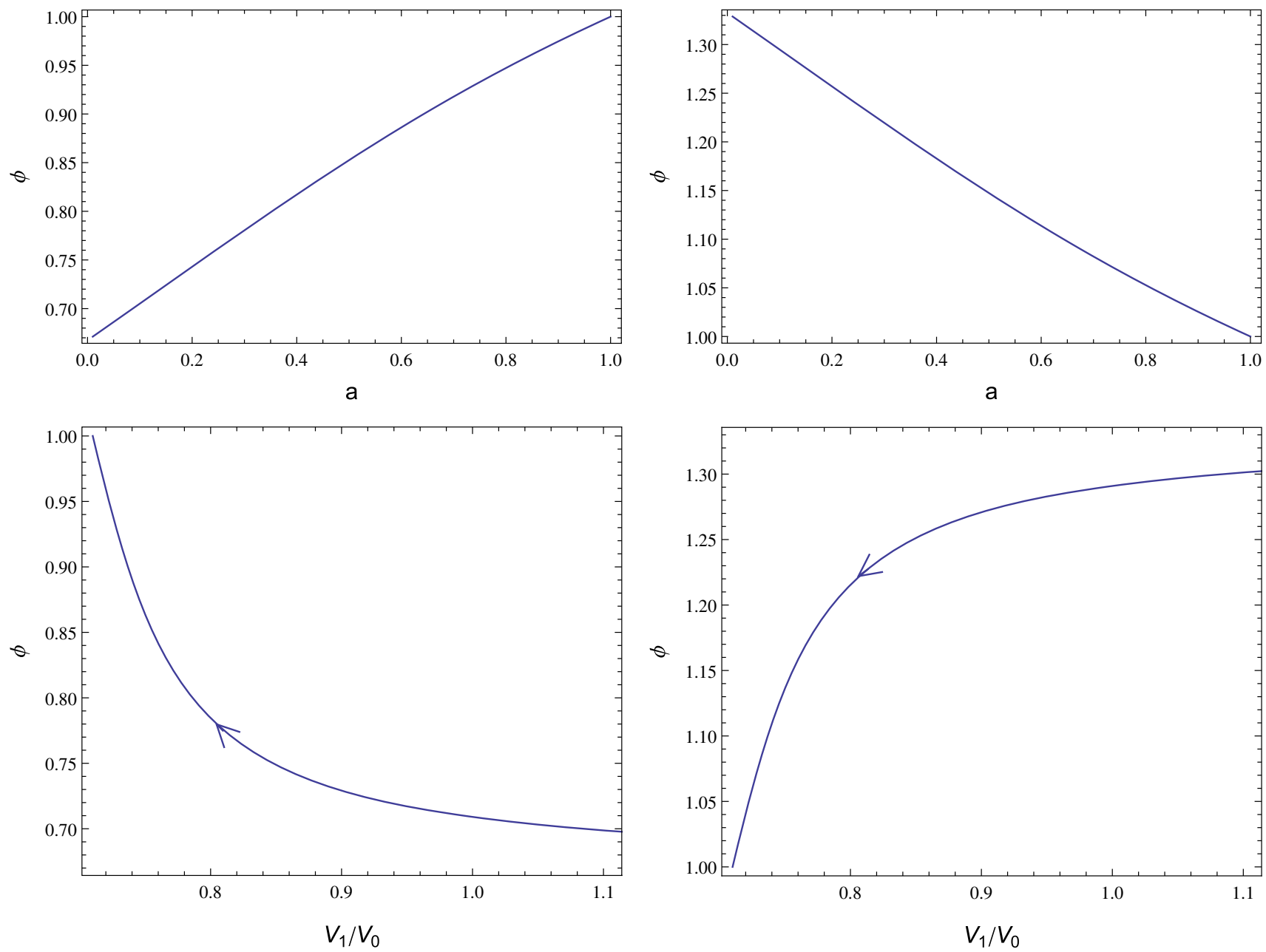

Fig. 1 The upper left andlower left panels corresponding to a plus sign in Eq. (22) represent the relation between the quintessence field $\phi$ and the scale factor $a$, and the relation between the quintessence field $\phi$ and the potential $V_{1} / V_{0}$. The upper right and lower right panels corresponding to a minus sign in Eq. (22) represent the relation between the

implies the Universe will eventually tend to be a de Sitter one. In the upper right panel of Fig. 1, one can find $\phi$ decreases with the increasing $a$, and in the lower right panel of Fig. 1, the potential decreases monotonically with the decreasing $\phi$ and also reaches its minimal value at the recent future. Furthermore, using Eq. (4), we can obtain the density ratio parameter $\Omega_{\phi}$ of the quintessence field as

$\Omega_{\phi}=\frac{\alpha+\beta\left(2 a^{-1}-a\right)}{\alpha+\beta\left(2 a^{-1}-a\right)+(1-\alpha-\beta) a^{-3}}$.

The evolutional tendency of the density ratio parameter $\Omega_{\phi}$ of the quintessence field is exhibited in Fig. 2, which is cosmologically dominant until low redshifts and is well consistent with the $\Lambda \mathrm{CDM}$ model at low redshifts.

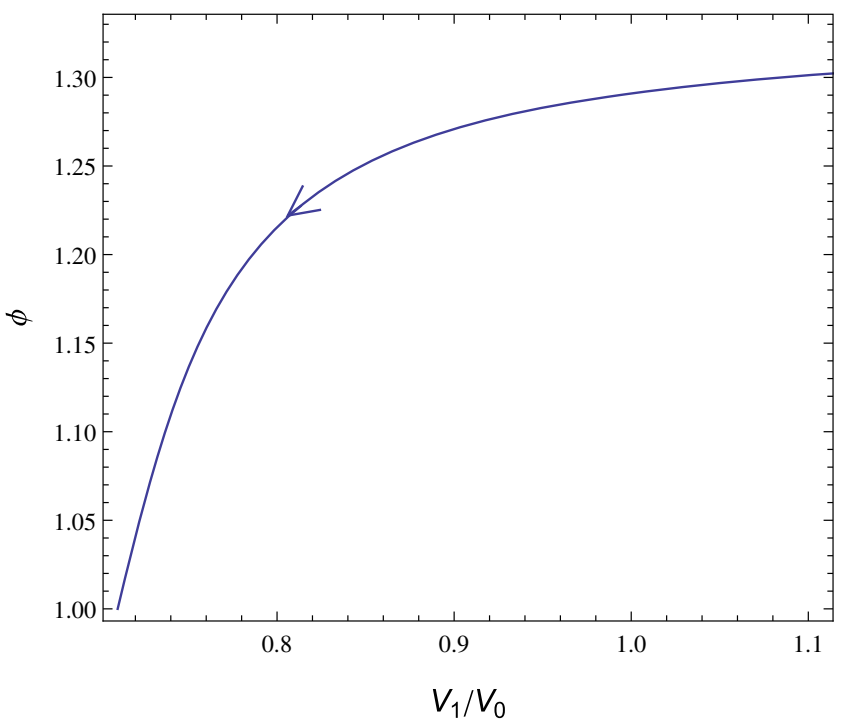

quintessence field $\phi$ and the scale factor $a$, and the relation between the quintessence field $\phi$ and the potential $V_{1} / V_{0}$. The arrows indicate the evolutional direction of the potential. We have assumed $\alpha=0.7$ and $\beta=0.02$ numerically

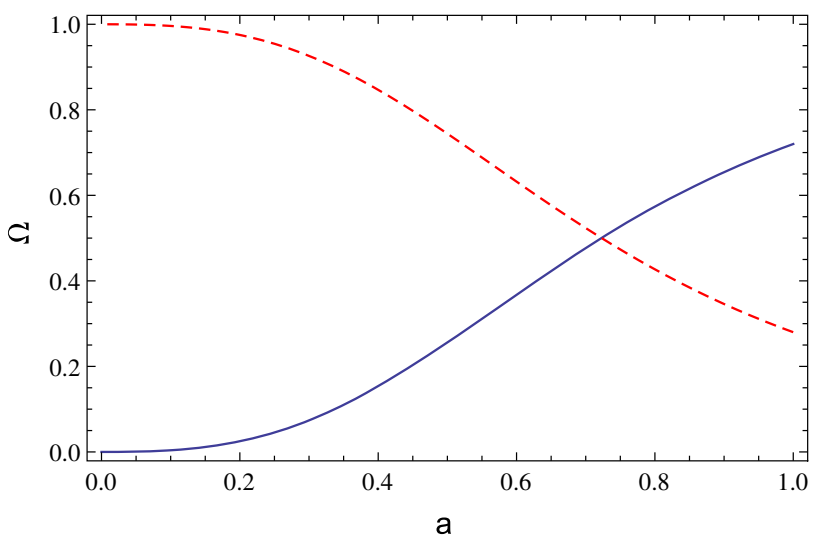

Fig. 2 The evolutional behaviors of the density ratio parameters for the quintessence field (solid line) and the matter (dashed line) 


\subsection{The phantom case}

Utilizing Eqs. (2), (3) and Eqs. (15), (16) and assuming the cosmic pie consists of two ingredients (phantom and matter), one can obtain

$$
\begin{aligned}
& -\frac{1}{2} \dot{\phi}^{2}+V_{2}(\phi)=-P_{a}+\frac{3}{4} P_{b}\left(a-2 a^{-1}\right), \\
& -\frac{1}{2} \dot{\phi}^{2}-V_{2}(\phi)=P_{a}+P_{b}\left(a^{-1}-a\right) .
\end{aligned}
$$

Similarly, simplifying the above two equations with the redefined parameters $\alpha$ and $\beta$, we have

$\frac{1}{2} \dot{\phi}^{2}=-\frac{1}{6} \rho_{0} \beta\left(a+2 a^{-1}\right)$,

$V_{2}(\phi)=\rho_{0}\left[\alpha+\frac{1}{6} \beta\left(10 a^{-1}-7 a\right)\right]$.

Utilizing Eq. (27), we find the free parameter $\beta<0$. Combining Eqs. (27) and (28) with the model parameters $\alpha$ and $\beta$, we obtain the following two equations:

$$
\frac{\mathrm{d} \phi}{\mathrm{d} a}= \pm M_{\mathrm{pl}}\left[-\frac{\beta\left(a^{-1}+2 a^{-3}\right)}{\alpha+\beta\left(2 a^{-1}-a\right)+(1-\alpha-\beta) a^{-3}}\right]^{\frac{1}{2}},
$$

$V_{2}(\phi)=V_{0}\left[\alpha+\frac{1}{6} \beta\left(10 a^{-1}-7 a\right)\right]$.

According to Eq. (24), here we explore the evolutional behaviors of the quintessence fields numerically by considering $\alpha=0.7$ and $\beta=-0.02$. In the upper left panel of Fig. 3, one can find that the phantom field $\phi$ increases monotonically with $a$, and that in the lower left panel of Fig. 3, the potential increases monotonically with the increasing $\phi$ and it will reach its maximal value at the recent future. From the upper right panel of Fig. 3, one can find $\phi$ decreases with the increasing $a$, and in the lower right panel of Fig. 3 , the potential increases monotonically with the decreasing $\phi$ and also reaches its maximal value at the recent future. This implies that the phantom field will come to rest at the maximum of the potential and the Universe tends to be a de Sitter one. By investigating the evolutional tendency of the density ratio parameter, we find the phantom field is cosmologically dominant at low redshifts and is well consistent with the predictions of the $\Lambda \mathrm{CDM}$ model (Fig. 4).

\section{The constraints}

In this section, we adopt the Markov Chain Monte Carlo (MCMC) method to constrain the pressure-parametrization UDF model by using SNe Ia, BAO and CMB observations.
We use the publicly available package CosmoMC $[47,48]$ to estimate the cosmological parameters that describe the data best. In order to obtain converging results, we stop sampling by checking the worst e-values (the variance of chain means/mean of variances) and $R-1$ is of order 0.01 in the MCMC calculations.

The observations of SNe Ia provide a powerful tool to probe the expansion history of the Universe. As is well known, the absolute magnitudes of all the SNe Ia are considered to be the same, since all the $\mathrm{SNe}$ Ia almost explode at the same mass $(M \approx-19.3 \pm 0.3)$. In light of this reasoning, $\mathrm{SNe}$ Ia can theoretically be used as the standard candles to constrain different cosmological models. In this analysis, we use the "Joint Light-curve Analysis" (JLA) containing $740 \mathrm{SNe}$ Ia data points, which covers the redshift range $z \in[0.01,1.3]$ [49]. JLA data can be divided into four classes: 118 low- $z$ $\mathrm{SNe}$ in the range $z \in[0,0.1]$ from [50-55]; $374 \mathrm{SNe}$ in the range $z \in[0.3,0.4]$ from the Sloan Digital Sky Survey (SDSS) SNe search [56]; $239 \mathrm{SNe}$ in the range $z \in[0.1,1.1]$ from the Supernova Legacy Survey (SNLS) project [57]; 9 high- $z$ SNe in the range $z \in[0.8,1.3]$ from the Hubble Space Telescope (HST) [58].

The baryon acoustic oscillations in the primordial plasma have striking effects on the anisotropies of the CMB and the large-scale structure of matter. We also use four different BAO measurements: the 6dFGS at effective redshift $z_{e f f}=$ 0.106 [59], the SDSS-MGS at $z_{\text {eff }}=0.15$ [60], the BOSSLOWZ at $z_{e f f}=0.32$ and the CMASS-DR 11 at $z_{\text {eff }}=0.57$ [61].

As is well known, the afterglow of the big bang-the $\mathrm{CMB}$ - not only provides some useful information on the very early Universe, but it also can give information on the expansion history of the Universe through the positions of the CMB acoustic peaks. In our analysis, we also use the CMB data from Planck-2015 [62], i.e., a joint observations of lensing + lowTEB + low $l$ TT temperature fluctuations angular power spectrum (see the online Package [63]).

To perform the so-called $\chi^{2}$ statistics, we choose the total likelihood function $\mathcal{L} \propto \mathrm{e}^{-\chi^{2} / 2}$ as the product of the separate likelihoods of SNe Ia, BAO and CMB data. Then the total $\chi^{2}$ can be expressed as

$\chi^{2}=\chi_{\mathrm{SNe}}^{2}+\chi_{\mathrm{BAO}}^{2}+\chi_{\mathrm{CMB}}^{2}$,

with the corresponding six-dimensional parameter space

$\left\{100 \theta_{\mathrm{MC}}, \tau, \alpha, \beta, \ln \left(10^{10} A_{s}\right), n_{s}\right\}$,

where $\tau$ is the Thomson scattering optical depth due to reionization, $\ln \left(10^{10} A_{s}\right)$ and $n_{s}$ are the amplitude and the spectral index of the primordial scalar perturbation power spectrum for the pivot scale $K_{0}=0.05 \mathrm{Mpc}^{-1}$, and $100 \theta_{\mathrm{MC}}$ denotes the approximation to $100 \times$ angular size of sound horizon at the redshift of last scattering $z_{*}$. To implement the con- 

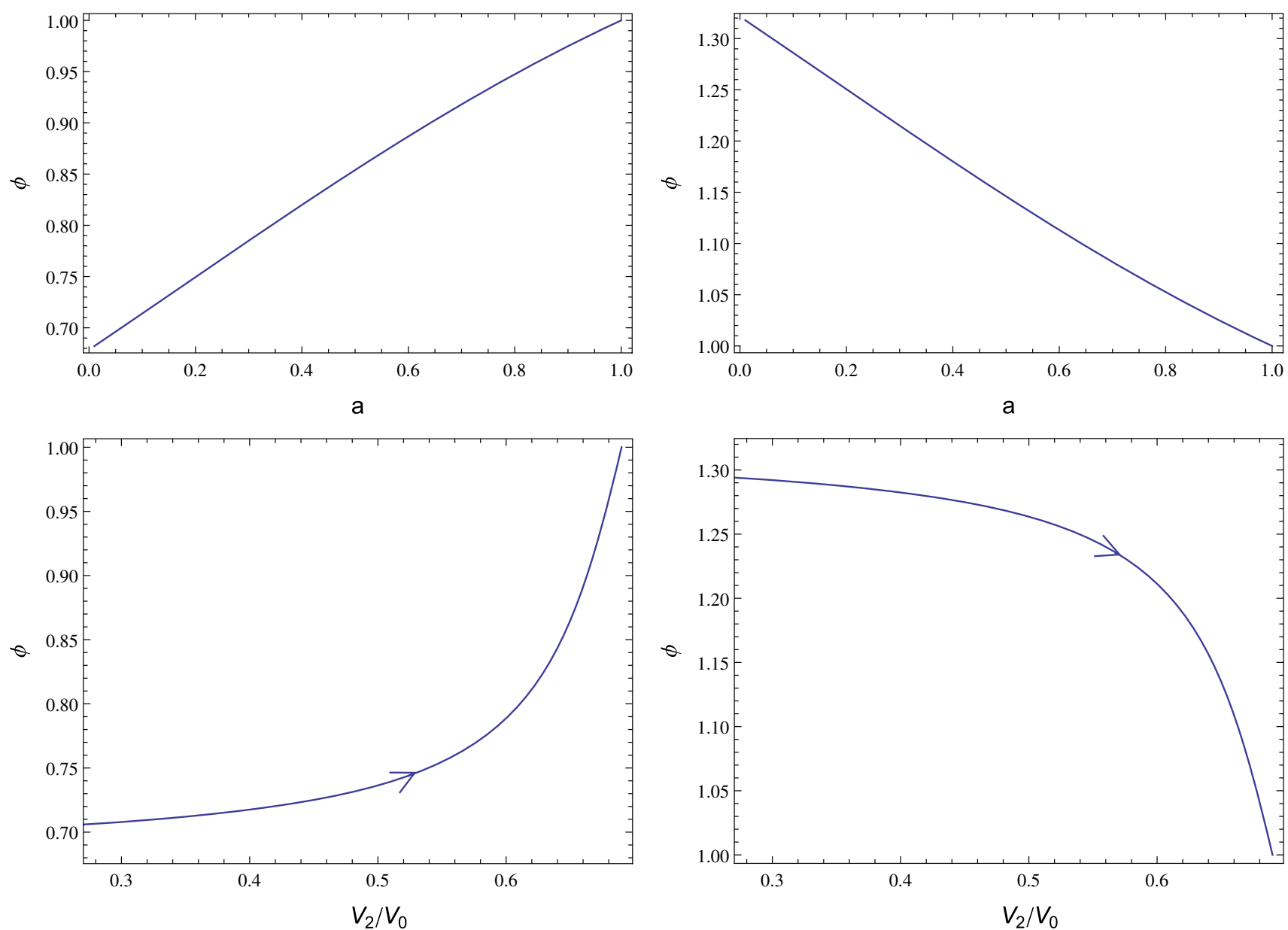

Fig. 3 The upper left and lower left panels corresponding to a plus sign in Eq. (29) represent the relation between the phantom field $\phi$ and the scale factor $a$, and the relation between the phantom field $\phi$ and the potential $V_{2} / V_{0}$. The upper right and lower right panels corresponding to a minus sign in Eq. (29) represent the relation between the phantom

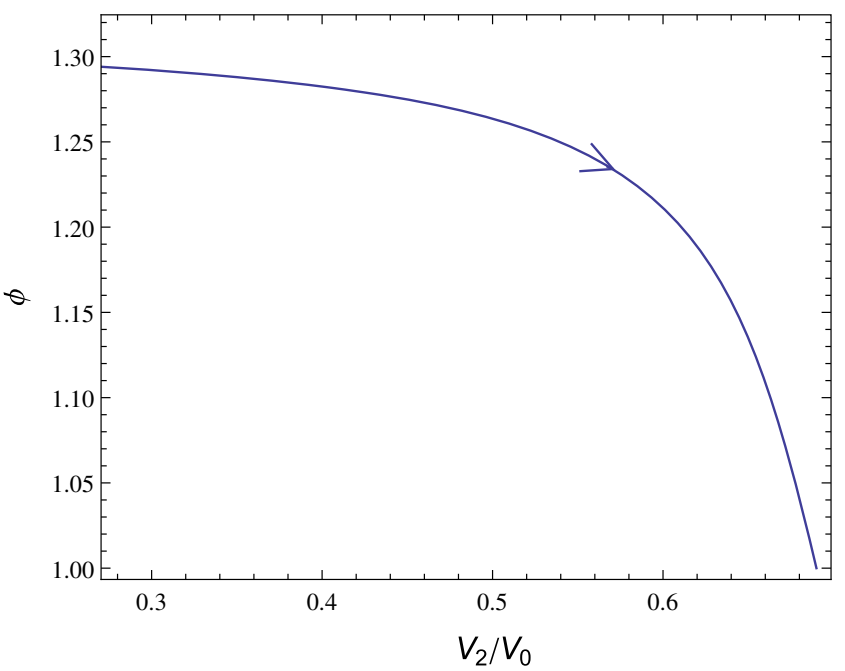

field $\phi$ and the scale factor $a$, and the relation between the phantom field $\phi$ and the potential $V_{2} / V_{0}$. The arrows indicate the evolutional direction of the potential. We have assumed $\alpha=0.7$ and $\beta=-0.02$ numerically

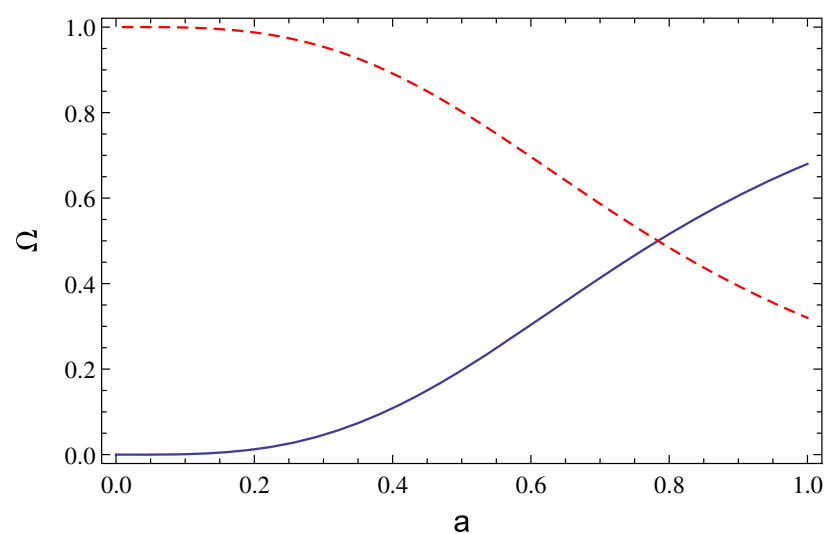

Fig. 4 The evolutional behaviors of the density ratio parameters for the phantom field (solid line) and the matter (dashed line)

straints, we list the priors on the parameter space in Table 1. In Fig. 5, we exhibit the one-dimensional marginalized probability distribution on the individual parameter and two-
Table 1 The priors of different parameters used in the posterior analysis

\begin{tabular}{ll}
\hline Parameter & Prior \\
\hline $100 \theta_{\mathrm{MC}}$ & {$[0.5,10]$} \\
$\tau$ & {$[0.01,0.8]$} \\
$\alpha$ & {$[0.5,0.9]$} \\
$\beta$ & {$[-0.1,0.1]$} \\
$\ln \left[10^{10} A_{s}\right]$ & {$[2.0,4.0]$} \\
$n_{s}$ & {$[0.8,1.2]$} \\
$H_{0}$ & {$[40,100]$} \\
\hline
\end{tabular}

dimensional contours of our UDF model by using $\mathrm{SNe}+$ $\mathrm{BAO}+\mathrm{CMB}$ observations. Meanwhile, the best-fit value and $1 \sigma$ error of the model parameters are presented in Table 2. One can find that the value of $\alpha$ is about $\alpha \sim 0.7$, which approaches the contribution of the $\mathrm{CC}$ in the $\Lambda \mathrm{CDM}$ model, and the value of $\beta$ is constrained to be very small. This implies our UDF model deviates slightly from the $\Lambda$ CDM model in a point of view of data. 


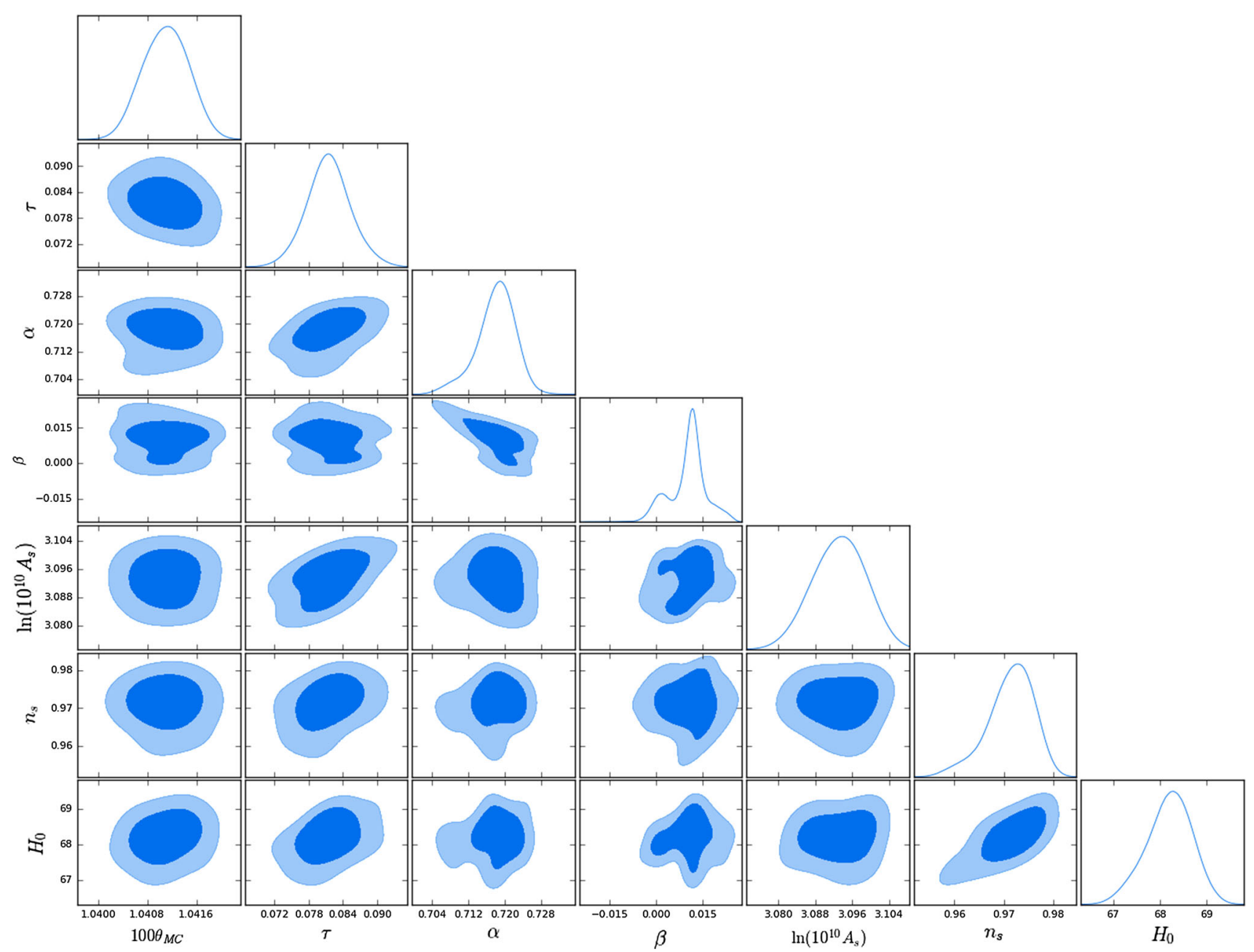

Fig. 5 The one-dimensional marginalized probability distribution on the individual parameter and two-dimensional contours of our UDF model by using $\mathrm{SNe}+\mathrm{BAO}+\mathrm{CMB}$ observations

\section{The diagnostics}

In this section, we are full of interest to distinguish the new UDF model from the $\Lambda$ CDM model by using the DE $\operatorname{EoS} \omega(a)$, deceleration parameter $q(a)$ and so-called $O m(a)$ diagnostic [64]. The expression of the DEEoS $\omega(a)$ is shown in Eq. (5) and the deceleration parameter $q(a)$ is written as

$q(a)=-\frac{a}{E(a)} \frac{\mathrm{d} E(a)}{\mathrm{d} a}-1$,

where $E(a)$ of the model is shown in Eq. (4).

The $\operatorname{Om}(a)$ diagnostic is an useful method to distinguish various kinds of cosmological models from the $\Lambda \mathrm{CDM}$ model and one from the other, which can be defined as

$O m(a)=\frac{E^{2}(a)-1}{a^{-3}-1}$.

It is easy to see that the $\operatorname{Om}(a)$ diagnostic involves only the first derivative of the scale factor through the Hubble
Table 2 The best-fit values and $1 \sigma$ errors of different parameters from the joint constraints $\mathrm{SNe}+\mathrm{BAO}+\mathrm{CMB}$

\begin{tabular}{ll}
\hline Parameter & Best-fit value with $1 \sigma$ error \\
\hline $100 \theta_{\mathrm{MC}}$ & $1.0411_{-0.0007}^{+0.0006}$ \\
$\tau$ & $0.082_{-0.007}^{+0.005}$ \\
$\alpha$ & $0.718_{-0.005}^{+0.004}$ \\
$\beta$ & $0.011_{-0.013}^{+0.006}$ \\
$\ln \left[10^{10} A_{s}\right]$ & $3.093_{-0.008}^{+0.007}$ \\
$n_{s}$ & $0.973_{-0.007}^{+0.005}$ \\
$H_{0}$ & $68.34_{-0.92}^{+0.53}$ \\
\hline
\end{tabular}

parameter and is easier to reconstruct from the observed data. For a spatially flat $\Lambda \mathrm{CDM}$ model, it is simply

$\operatorname{Om}(a)=\Omega_{m 0}$,

where $\Omega_{m 0}$ denotes the present-day value of the matter density ratio parameter. 

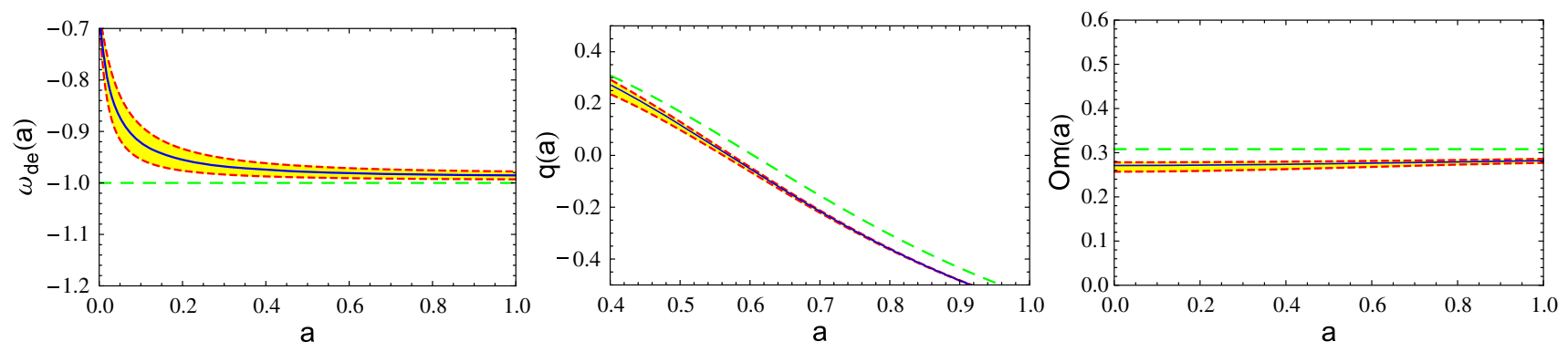

Fig. 6 From left to right, we exhibit the relations between the scale factor $a$ and the DE $\operatorname{EoS} \omega(a)$, deceleration parameter $q(a)$ and $\mathrm{Om}(\mathrm{a})$ diagnostic, respectively. The solid (blue) lines and long-dashed (green) lines correspond to the pressure-parametrization UDF model

Using $\Omega_{m 0}=0.308$ [62] for the flat $\Lambda$ CDM model and error propagations of the model parameters $(\alpha, \beta)$, we exhibit the above three diagnostics of the UDF model in Fig. 6. One can find that the UDF model deviates slightly from the $\Lambda \mathrm{CDM}$ model at low redshifts and it will approach gradually the $\Lambda \mathrm{CDM}$ model in the future. This indicates that the small deviation from the $\Lambda$ CDM model has been realized by our new UDF model, and it may provide a new possibility to explain the puzzles, which the standard cosmology meets.

\section{The $H_{0}$ tension}

Recently, the improved local measurement $H_{0}=73.24 \pm$ $1.74 \mathrm{~km} \mathrm{~s}^{-1} \mathrm{Mpc}^{-1}$ from Riess et al. 2016 (hereafter R16) [65] exhibits a stronger tension with the Planck 2016 release $H_{0}=66.93 \pm 0.62 \mathrm{~km} \mathrm{~s}^{-1} \mathrm{Mpc}^{-1}$ (hereafter P15) [66] at the $3.4 \sigma$ level. In this analysis, we try to explain it by utilizing the new UDF model deviating slightly from the $\Lambda$ CDM model. We obtain $H_{0}=68.34_{-0.92}^{+0.53}$, which is consistent with the P15's result at the $1 \sigma$ level (see Fig. 5; Table 2).

\section{Discussions and conclusions}

Parametrization approaches for the effective pressure of the dark contents of the Universe are aimed at exploring various kinds of possible small deviations from the $\Lambda \mathrm{CDM}$ model, in order to alleviate or even solve the problems which exist in the $\Lambda \mathrm{CDM}$ model. Comparing with the parametrization approaches for the DE EoS, it is interesting to investigate the deviations from the constant $P(z)-z$ relation without any prejudice to a presupposition.

In this work, we propose a new pressure-parametrization model by regarding the baryon matter and dark contents of the Universe as a unified dark fluid. To realize the new UDF model more physically, we reconstruct it with the quintessence and phantom scalar fields, and we find that the Universe eventually tends to be of de Sitter type. Subsequently, we constrain the UDF model using the SNe Ia, BAO and $\Lambda \mathrm{CDM}$ model, respectively. The shaded (yellow) regions and shortdashed (red) lines represent the $1 \sigma$ regions and corresponding boundaries

and $\mathrm{CMB}$ observations, and we find that the value of $\alpha$ is about $\alpha \sim 0.7$, which approaches the contribution of the $\mathrm{CC}$ in the $\Lambda \mathrm{CDM}$ model, and the value of $\beta$ is constrained to be very small. This implies that our UDF model deviates slightly from the $\Lambda \mathrm{CDM}$ model in a point of view of data. Furthermore, utilizing the constrained model parameters $(\alpha, \beta)$, we distinguish the UDF model from the $\Lambda \mathrm{CDM}$ model by the DE EoS $\omega(a)$, deceleration parameter $q(a)$ and so-called $O m(a)$ diagnostic. We find that the UDF model deviates slightly from the $\Lambda \mathrm{CDM}$ model at low redshifts and it will approach gradually the $\Lambda \mathrm{CDM}$ model in the future. This indicates that the small deviation from the $\Lambda \mathrm{CDM}$ model has been realized by our new UDF model, and it may provide a new possibility to explain the problems and puzzles, which the $\Lambda \mathrm{CDM}$ model meets. Specifically, the first two terms in Eq. (3) is actually equivalent to a dynamical CC $\Lambda(a)$, to some extent, which could help us to resolve the fine-tuning problem (see [67] for details) and current $H_{0}$ anomaly. It is worth noting that some authors also suggested that a dynamical $\mathrm{CC}$ is the requirement of the laws of quantum field theory (see [68] for a recent review).

Using the joint constraints $\mathrm{SNe}+\mathrm{BAO}+\mathrm{CMB}$, we also try to explain the current $H_{0}$ tension by utilizing the UDF model deviating slightly from the $\Lambda \mathrm{CDM}$ model. The value of the Hubble constant $H_{0}=68.34_{-0.92}^{+0.53}$ is consistent with the P15's result at the $1 \sigma$ confidence level (see Fig. 5; Table 2).

In a follow-up study, we explore some possible parametriof alleviating the puzzles which the $\Lambda \mathrm{CDM}$ model meets. For example, (i) $P(z)=P_{a}+P_{b} \ln (1+z)$ : this model leads to a small logarithmic deviation from the $\Lambda \mathrm{CDM}$ model; (ii) $P(z)=P_{a}+P_{b} \frac{z(1+z)}{1+z^{2}}$ or $P(z)=P_{a} \sin \left(\frac{1}{1+z}\right)$ : interesting periodic deviations occur in these two models; (iii) $P(z)=P_{a}+P_{b}(1+z)^{2}+P_{c}(1+z)^{4}:$ it is interesting that the $\Lambda$ CDM model will be recovered in this model, containing four different evolution epochs of the Universe. The detailed analyses of these pressure-parametrization UDF models are in preparation. zation models for the effective pressure and test their abilities 
Acknowledgements This study is supported in part by the National Science Foundation of China. We thank Profs. S. D. Odintsov and Bharat Ratra for useful communications on cosmology and theories of gravity. Deng Wang warmly thanks Prof. Jing-Ling Chen for beneficial discussions.

Open Access This article is distributed under the terms of the Creative Commons Attribution 4.0 International License (http://creativecomm ons.org/licenses/by/4.0/), which permits unrestricted use, distribution, and reproduction in any medium, provided you give appropriate credit to the original author(s) and the source, provide a link to the Creative Commons license, and indicate if changes were made. Funded by SCOAP ${ }^{3}$.

\section{References}

1. A.G. Riess et al., Supernova Search Team, Astron. J. 116, 1009 (1998)

2. S. Perlmutter et al., Supernova Cosmology Project, Phys. Rev. Lett. 83, 670 (1999)

3. P. Ade et al., Planck Collaboration, Astron. Astrophys. 571, A16 (2014)

4. D.H. Weinberg et al., Phys. Rep. 530, 87 (2013)

5. S.M. Carroll, Living Rev. Relativ. 4, 1 (2001)

6. S. Weinberg, Rev. Mod. Phys. 61, 1 (1989)

7. R.R. Caldwell, Phys. Lett. B 545, 23-29 (2002)

8. Y. Fujii, Phys. Rev. D 26, 2580 (1982)

9. L.H. Ford, Phys. Rev. D 35, 2339 (1987)

10. C. Wetterich, Nucl. Phys. B 302, 668 (1988)

11. B. Ratra, P.J.E. Peebles, Phys. Rev. D 37, 3406 (1988)

12. S.M. Carroll, Phys. Rev. Lett. 81, 3067 (1998)

13. A. Hebecker, C. Witterich, Phys. Rev. Lett. 86, 3339 (2000)

14. A. Hebecker, C. Witterich, Phys. Lett. B 497, 281 (2001)

15. R.R. Caldwell, M. Kamionkovski, N.N. Weinberg, Phys. Rev. Lett. 91, 071301 (2003)

16. X.-H. Meng, J. Ren, M.G. Hu, Commun. Theor. Phys. 47, 379 (2007). arXiv:astro-ph/0509250

17. J. Ren, X.-H. Meng, Phys. Lett. B 636, 5 (2006)

18. J. Ren, X.-H. Meng, Phys. Lett. B 633, 1 (2006)

19. M.G. Hu, X.-H. Meng, Phys. Lett. B 635, 186 (2006)

20. X.-H. Meng, X. Dou, Commun. Theor. Phys. 52, 377 (2009)

21. X. Dou, X.-H. Meng, Adv. Astron. 1155, 829340 (2011)

22. J.A.S. Lima, Phys. Rev. D 54, 2571 (1996)

23. J.A.S. Lima, J.S. Alcaniz, Astron. Astrophys. 348, 1 (1999)

24. P. Wang, X.-H. Meng, Class. Quant. Grav. 22, 283-294 (2005)

25. A. Kamenshchik, U. Moschella, V. Pasquier, Phys. Lett. B 511, $265(2001)$
26. S. Capozziello, Int. J. Mod. Phys. D 11, 483 (2002)

27. S. Capozziello et al., Int. J. Mod. Phys. D 12, 1969 (2003)

28. S.M. Carroll et al., Phys. Rev. D 70, 043528 (2004)

29. S. Nojiri, S.D. Odintsov, Phys. Rev. D 68, 123512 (2003)

30. S. Nojiri, S.D. Odintsov, Phys. Rep. 505, 59-144 (2011)

31. S. Nojiri, S.D. Odintsov, Phys. Lett. B 681, 74-80 (2009)

32. T. Jacobson, Einstein-Aether gravity: a status report, PoS QG-PH: 020 (2007)

33. T. Jacobson, Phys. Rev. D 81, 101502 (2010)

34. L. Randall, R. Sundrum, Phys. Rev. Lett. 83, 3370 (1999)

35. L. Randall, R. Sundrum, Phys. Rev. Lett. 83, 4690 (1999)

36. G.R. Davli, G. Gabadadze, M. Porrati, Phys. Lett. B 485, 208 (2000)

37. D. Huterer, M.S. Turner, Phys. Rev. D 60, 081301 (1999)

38. J. Weller, A. Albrecht, Phys. Rev. D 65, 103512 (2002)

39. M. Chevallier, D. Polarski, Int. J. Mod. Phys. D 10, 213 (2001)

40. E.V. Linder, Phys. Rev. Lett. 90, 091301 (2003)

41. G. Pantazis et al., Phys. Rev. D 93, 103503 (2016)

42. G. Efstathiou, MNRAS 310, 842 (1999)

43. E.M. Barboza Jr. et al., Phys. Lett. B 666, 415-419 (2008)

44. H. Wei et al., JCAP 01, 045 (2014)

45. A.A. Sen, Phys. Rev. D 77, 043508 (2008)

46. Q. Zhang, G. Yang, Q.-X. Zou, X.-H. Meng, Ke-Ji Shen, Eur. Phys. J. C 75, 300 (2015)

47. A. Lewis, A. Challinor, A. Lasenby, Astrophys. J. 538, 473 (2000)

48. A. Lewis, S. Bridle, Phys. Rev. D 66, 103511 (2002)

49. M. Betoule et al., SDSS Collaboration. Astron. Astrophys. 568, A22 (2014). arXiv: 1401.4064

50. M. Hamuy et al., Astron. J. 112, 2408 (1996)

51. A.G. Riess et al., Astron. J. 117, 707 (1999)

52. S. Jha et al., Astron. J. 131, 527 (2006)

53. C. Contreras et al., Astron. J. 139, 519 (2010)

54. M. Hicken et al., Astrophys. J. 700, 1097 (2009)

55. M. Hicken et al., Astrophys. J. 700, 331 (2009)

56. J. Holtzman et al., Astron. J. 136, 2306 (2008)

57. J. Guy et al., A \& A 523, 7 (2010)

58. A.G. Riess et al., Astrophys. J. 659, 98 (2007)

59. F. Beutler et al., Mon. Not. R. Astron. Soc. 3017, 416 (2011)

60. A.J. Ross et al., Mon. Not. R. Astron. Soc. 835, 449 (2015)

61. L. Anderson et al., BOSS collaboration, Mon. Not. R. Astron. Soc. 24, 441 (2014)

62. Planck Collaboration, A \& A 594, A13 (2016)

63. Cosmological MonteCarlo. Web page maintained by Antony Lewis. http://cosmologist.info/cosmomc/. Accessed 2 June 2015

64. M. Arabsalmani, V. Sahni, Phys. Rev. D 86, 103527 (2012)

65. A.G. Riess et al., Astrophys. J. 826, 1 (2016)

66. N. Aghanim et al., Planck Collaboration, A \& A 596, A107 (2016)

67. M. Bronstein, Phys. Z. Sowjetunion 3, 73 (1933)

68. T. Padmanabhan, Phys. Rep. 380, 235 (2003) 